"Proof in the Wording: Two modalities from Ancient Chinese Algorithms", in G. Hanna, H. N. Jahnke, H. Pulte, Explanation and Proof in Mathematics: Philosophical and Educational Perspectives, Springer, 2010, pp. 253-285.

\title{
Chapter 17 \\ Proof in the Wording: Two Modalities from Ancient Chinese Algorithms
}

\author{
Karine Chemla
}

\subsection{Introduction}

The earliest extant Chinese mathematical documents do not contain theorems, but rather algorithms, most of which - though not all - were presented in relation to problems. This holds true for writings that came down to us through two different channels. Some of these writings are known only through manuscripts excavated in the twentieth century from tombs in which, in the last centuries B.C.E, they had been buried with their owners. This is the case with the Book of Mathematical Procedures (算數書, Suanshushu), found in 1984 in a tomb sealed before circa 186 B.C.E. ${ }^{1}$ Other writings were handed down through the written tradition, for example, The Nine Chapters on Mathematical Procedures (九章算術, Jiuzhang suanshu), which dates to the first century C.E. ${ }^{2}$ Two early commentaries on The Nine Chapters were also handed down together with it until today. In fact, there is no ancient edition of The Nine Chapters that would not contain the commentary completed by Liu Hui (劉徽) in 263 or the supra-commentary on the two layers of text presented to the throne in 656 and composed by a group of scholars led by Li Chunfeng (李淳風). ${ }^{3}$

\footnotetext{
${ }^{1}$ Compare the critical edition with annotations in Peng Hao (彭浩 2001).

${ }^{2}$ Below, I shall abbreviate the title into The Nine Chapters. For a critical edition and a French translation of this book and its earliest commentaries, compare Chemla and Guo Shuchun 2004. Chapter B, by Guo Shuchun, discusses the opinions of several scholars regarding the time period when The Nine Chapters was compiled. In my introduction to chapter 6 in the same book, I argue for dating the end of the compilation to the first century C.E. (Chemla and Guo Shuchun 2004: 475-481).

3 Below, we refer to this layer of the text as "Li Chunfeng's commentary." Two other supracommentaries, composed during the Song dynasty, respectively in the eleventh and the thirteenth century, survived only partially. They were not handed down systematically with the collection, by that time coherent, that The Nine Chapters and the two earlier commentaries formed.
} 
As a consequence, in these writings, mathematical proofs did not take the form of proofs of the truth of theorems but rather that of proofs of the correctness of algorithms. Whether the algorithms related to geometrical, algebraic or arithmetical questions, the proofs established that both the meaning of the result and the value yielded corresponded to the magnitude sought. ${ }^{4}$ Hence, the texts give us an opportunity to think about proofs of the correctness of algorithms, a kind of proof so far seldom examined in discussions about mathematical proof. ${ }^{5}$

What kind of evidence do we have in these ancient Chinese writings regarding such proofs? The commentaries that Liu Hui and Li Chunfeng developed in relation to virtually every procedure of The Nine Chapters systematically established the correctness of the procedures. They provide ample evidence with respect to how such a proof was conducted; they have been abundantly studied in the past decades. ${ }^{6}$ However, the two commentaries indicate another type of evidence, more complex from a methodological point of view. Recently, I have been struggling with the idea that the commentators were sometimes "reading" their proofs in the way in which the texts for the algorithms were formulated in The Nine Chapters. ${ }^{7}$ In fact, many hints indicate that The Nine Chapters regularly pointed out reasons for which the algorithms were correct in the very way in which the text for the algorithms in the book was written. This feature reveals that the relationship between the text of an algorithm and the text of a proof of its correctness is not as simple as we spontaneously assume. This issue is in fact part of a wider problem: namely, how the text of an algorithm is handled when the question of its correctness is addressed. For lack of space, I cannot deal systematically with the wider problem here. Rather, I shall concentrate on the question of how the text of an algorithm can in and of itself indicate reasons for that algorithm's correctness. The question is essential to address, if we want to delineate the evidence from ancient China on the basis of which to examine the history of the ways by which the correctness of an algorithm was addressed. The evidence from ancient China provides abundant source material to ponder with a certain generality the issue raised with respect to texts. In this paper, I shall concentrate on this evidence

\footnotetext{
${ }^{4}$ I introduced this distinction in Chemla 1996. I shall come back to it below.

${ }^{5}$ More precisely, when such proofs were analyzed, their analysis seldom aimed at determining the specificities of proofs, whose goal is to establish the correctness of algorithms. I have suggested elsewhere that once we understand better the history of such proofs, we might be in a position to formulate hypotheses regarding the part they played in a world history of mathematical proof and, more specifically, in a history of algebraic proof. However, in my view, we have not yet reached that point.

${ }^{6}$ It would be impossible to mention here the many papers and books that in the last decades were devoted to the proofs contained in the commentaries. Let me simply evoke: Li Yan (李儼 1958: 4054); Qian Baocong (錢寶琮 1964: 62-72); Wu Wenjun (㺯文俊 1982), Li Jimin (李繼閔 1990); Guo Shuchun (郭書春 1992); Wu Wenjun (吳文俊), Bai Shangshu (白尚恕), Shen Kangshen (沈康身) and Li Di (李迪 1993). For a fuller bibliography, refer to Chemla and Guo Shuchun 2004. In general, the publications seldom analyze the proofs from the viewpoint that they establish the correctness of algorithms. I have attempted to identify the main operations involved in the proof of the correctness of algorithms to which these commentaries bear witness in Chap. A of Chemla and Guo Shuchun 2004: 27-39.

${ }^{7}$ The first synthetical article that I devoted to this issue is Chemla 1991.
} 
to clarify what it means that the text of an algorithm refers to a proof of its correctness.

With this perspective in mind, I shall begin by briefly reexamining some source material from The Nine Chapters and its commentaries that I have analyzed in previous publications. ${ }^{8}$ I shall then be in a position to illuminate two main families of techniques through which the text for an algorithm can refer to reasons for its correctness. Finally, I shall rely on this analysis to examine, from the same viewpoint, source material from the Book of Mathematical Procedures. Although the Book of Mathematical Procedures also makes use of the same two distinct kinds of techniques, the second technique is used differently than in The Nine Chapters and its commentaries. The final part of the article focuses on this latter technique, revealing similarities and differences in how, in these various writings, texts for algorithms refer to reasons for their correctness. The features examined thus help us bring to light differences between the two books that would remain unnoticed otherwise. Both those similarities and differences give clues to address an open question, that of the historical relationship between the Book of Mathematical Procedures, (recorded in a manuscript found in a tomb sealed at the beginning of the second century B.C.E.), and The Nine Chapters, (a book probably compiled in the first century C.E. and handed down). How can the differences highlighted between the two be accounted for? Do these differences indicate that these two writings emerged from distinct social milieus, or do they attest to an evolution in practice during the centuries between their composition. My analysis thus provides data that will help tackle the problem. Before we turn to considering these questions, however, some remarks on the text of an algorithm are in order.

\subsection{A Few Words on the Texts for Algorithms}

The problem of how the very text through which an algorithm is given refers to a proof of its correctness raises a fundamental issue, which we need to consider simultaneously: how does - or, more precisely, how did - one write a text for an algorithm? As Chinese sources illustrate, there are two types of reality corresponding to an algorithm. ${ }^{9}$

On the one hand, algorithms are given by means of texts recorded in books. These texts are commonly described as "sequences of operations." Moreover, they are usually qualified as "general," since they are valid not only for the problem in relation to which they are given, but for a class of similar problems. As a result, although at first sight they do look like "sequences of operations," we must be aware that the

\footnotetext{
${ }^{8}$ See Chemla 1991, 1996.

${ }^{9}$ The working seminar "History of science, history of text," organized with Jacques Virbel since 2002, and especially Agathe Keller's contribution, helped me clarify this dual dimension of an algorithm. It is my pleasure to express my gratitude to the group gathered around this seminar.
} 
textual appearance of the sequence sometimes hides complex structures in the list of operations. $^{10}$

On the other hand, there is usually, outside the book, an instrument for computing in ancient China, it was a surface on which numbers were written down with counting rods according to a place-value decimal system. On this instrument, the algorithm corresponds to actions performed, on actual values, transforming them until the result(s) appears. ${ }^{11}$ Below, I shall discuss this dimension of the algorithm mainly on the basis of the specific example of the surface used for computations in ancient China. I shall refer to this dimension, when seen from the point of view of the events occurring on the instrument, as the "flow of computations," thereby stressing that these actions form a sequence over time.

Usually, the text by means of which an algorithm is written down corresponds to several distinct lists of actions that can be taken on the instrument. Depending on the values to which the algorithm is applied and depending on the cases with which the practitioner is confronted, the single general text for the algorithm generates the various sequences of actions required. That the text giving an algorithm corresponds to distinct lists of actions raises the questions of how the text achieves the integration of these sequences of actions and how it corresponds to the various computational flows generated. Different textual solutions to those problems appear in various writings of the past, even if we restrict ourselves to Chinese sources. This remark reveals that the question of how the text giving an algorithm corresponds to distinct lists of actions has a less straightforward answer than may be spontaneously assumed.

The text for an algorithm can be analyzed from another angle. Usually, we do not have a one-to-one correspondence between the terms referring to operations in the text and the actions taken on the instrument. Suppose a multiplication is to be carried out. The text can either prescribe the operation by a term, which thus corresponds to a series of actions on the instrument, or embed the details of a procedure for multiplying. We shall refer to this distinction by introducing the concept of the "grain of the description": The grain can be finer or coarser, depending on whether actions on the instrument are grouped in operations at a higher level or not. We can analyze how a text for an algorithm carries out the regrouping of elementary actions by means of terms referring to operations from two perspectives. On the one hand, we can examine the way in which actions are grouped within a single operation. On the other hand, we can analyze the terms chosen to prescribe this operation. In relation to the fineness or the coarseness of the description and to how coarseness is achieved, the text for an algorithm can convey different ways of conceptualizing the various flows of computation for the function corresponding to the algorithm. We shall see below,

\footnotetext{
${ }^{10}$ See below for some concrete examples.

${ }^{11}$ I owe this element of description of an algorithm, that is, the "action," to the presentation of the project "Histoire de la calculabilité" by M. van Atten, M. Bourdeau, and J. Mosconi (Final Conference of the Program of the CNRS and MESR: "Histoire des savoirs," November 29-December 1, 2007). The proceedings of the Program can be found at http://www.cnrs.fr/prg/PIR/programmestermines/histsavoirs/synth2003-2007Histoiredessavoirs.pdf.
} 
without exhausting the variety of cases that can be documented from the Chinese sources, that several techniques were used to achieve that goal. This is precisely one aspect by means of which a text can indicate reasons of the correctness.

The use in a given text of terms referring to a single operation, for instance a multiplication, allows giving a single prescription for sequences of actions that may differ, depending on the values to be multiplied. This remark reveals a relationship between the two features of a text that we distinguished: a coarser grain in the details given by the text with respect to the sequence of actions to be executed is one means through which a single text allows handling different cases, though not the only one.

I now turn to some concrete texts for algorithms from The Nine Chapters and its earliest commentary. In addition to illustrating the distinctions just introduced, these texts will allow me to elucidate how the text for an algorithm can indicate reasons for its correctness.

\subsection{Texts for Algorithms - An Insight from The Nine Chapters}

\subsubsection{The Straightforward Reference to Operations and the Question of the Meaning}

The first example of a text for an algorithm is paradigmatic in two ways: On the one hand, it prescribes operations in a direct way. On the other hand, its structure allows that along the sequence of operations, step by step, sub-procedure by subprocedure, the meanings of the consecutive results are successively brought to light. Therefore, when the end of the text is reached, the meaning of the result can be made clear and can be shown to be precisely identical to that expected. It is thereby proved that the given algorithm yields the correct result.

In such types of texts for algorithms in The Nine Chapters, the commentator's proof amounts to establishing the meaning of the sequence of partial results until the end result is reached. ${ }^{12}$ The commentator thus in some sense reads a proof in the structure of the text. An excerpt that illustrates these phenomena is provided by the commentator Liu Hui. In it, Liu Hui writes down a text for an algorithm and at the same time, step by step, sub-procedure by sub-procedure, he provides an interpretation for each partial result. In some sense, he has merged the text of the algorithm and that of its proof into a single text. A formulation of that kind will make it easier for us to understand this type of text for algorithms and to suggest how these algorithms could be, on the one hand, obtained and, on the other hand, proved to be correct.

\footnotetext{
${ }^{12}$ I describe a text of that kind for an algorithm as well as Liu Hui's proof of the correctness of the algorithm in Chemla 1991.
} 
Our excerpt is the initial segment of an algorithm Liu Hui presents in his commentary after the first procedure given in The Nine Chapters to compute the area of a circle. ${ }^{13}$ In a passage preceding the one we shall analyze, Liu Hui had established the correctness of the algorithm stated in The Nine Chapters, which prescribed multiplying half of the diameter of the circle by half of its circumference to yield the area. He then exposes the fact that the ratio of 1-3 between these two data, which characterizes the values given in the statements of the problems in The Nine Chapters - the diameter and the circumference-,${ }^{14}$ differs from the one that the algorithm assumes if it is to be correct. Consequently, despite the correctness of the algorithm, the problems in The Nine Chapters do not provide values that guarantee the exactness of the result of the algorithm. In this context, Liu Hui sets out to compute other values. We shall examine the beginning of the text by means of which he writes down his algorithm.

First, I shall sketch out the idea of the computation, which Liu Hui bases upon the drawing he referred to in his proof of the correctness of the procedure given by The Nine Chapters (see Fig. 17.1). ${ }^{15}$ Liu Hui's whole text consists of the repetition of a

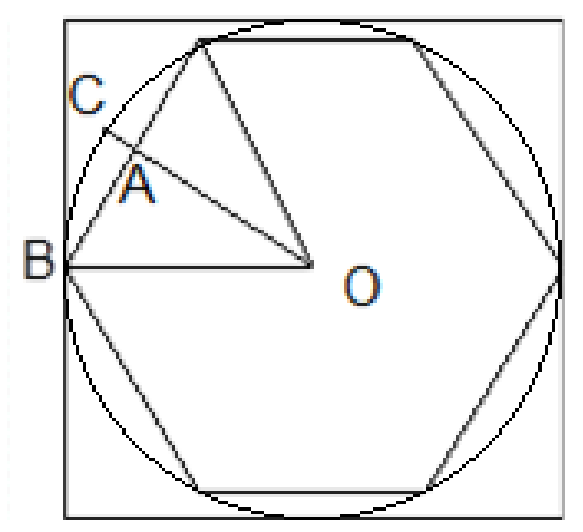

Fig. 17.1. The figure Liu Hui used to deal with the area of the circle

\footnotetext{
${ }^{13}$ I gave a more detailed analysis of the commentary on the area of the circle in Chemla 1996. For a critical edition and translation into French of the whole passage, see Chemla and Guo Shuchun 2004: 176-189.

${ }^{14}$ These are problems 1.31 and 1.32. The pair of numbers I attach to a given problem in The Nine Chapters refers, first, to the chapter in which it is placed (here, Chap. 1) and, then, to the order in which the problems are arranged in this chapter (here, 31st and 32nd problems). Note that these numbers are not part of the source material.

${ }^{15}$ Note that the diagram is restored on the basis of the references Liu Hui makes to its structure. However, I do not attempt to produce a figure conforming to the features known to be specific of the diagrams Liu Hui used. For instance, to conform to modern usage, I name some of the points. Before the thirteenth century C.E., we have no evidence in China of such ways of marking figures.
} 
sequence of operations which, from the point of view of the computations carried out, corresponds to the iteration of a procedure computing the length of the side of a regular $2 n$-gon inscribed into the circle, when knowing the length of the side of a $n$ gon and the diameter of the circle. Once he has reached the accuracy he looks for, Liu Hui derives from the side of the $n$-gon just computed the value of the circumference and hence the value of the area of the corresponding $2 n$-gon. I shall focus on the initial description of the sequence of operations, which starts from the side of the regular hexagon inscribed in the circle.

As is known from Liu Hui's previous proof in the commentary on the area of the circle, the side of a regular hexagon is equal to half of the diameter of the circle in which it is inscribed. The first half of the sequence of operations to be repeated makes use of the fact that in the right-angled triangle $\mathrm{OAB}$, both the base $(A B)$ and the hypotenuse $(O B)$ are known: they are, respectively, half of the side of the hexagon (more generally, the $n$-gon) and half of the diameter. Applying the "Pythagorean" procedure (the main topic of Chap. 9 in The Nine Chapters), one obtains the height $\mathrm{OA}$. Thereafter, in the right-angled triangle $\mathrm{ABC}$, given that the base is the difference between the radius and OA, and that the height is half the side of the $n$-gon, on the other hand, their values are known. In the second half of the procedure, applying again the "Pythagorean" procedure, one obtains CB, which is the side of the $2 n$-gon. One can then repeat the procedure to derive the length of the side of the $4 n$-gon, and so on.

Let us concentrate on how Liu Hui formulates this sequence of operations at the beginning of the excerpt. The first sentences of the procedure read as follows:

"Procedure consisting in cutting the 6-gon in order to make a 12-gon: One sets up the diameter of the circle, 2 chi. One halves it, which makes 1 chi and gives the side of the 6-gon that is in the circle,"

(割六触以爲十二觞術曰：置圓徑二尺, 半之爲一尺, 即圓裏触之面也。；my emphases).

The goal of the procedure is announced at the beginning of the text, in its name ("Procedure consisting in cutting the 6-gon in order to make a 12-gon"); the goal and the name - will change at each repetition of the sequence of operations, from $n$ gon and $2 n$-gon to, in the next step, $2 n$-gon and $4 n$-gon, and so on. In the initial procedure aiming to cut the hexagon into a 12-gon, the side of which is to be determined, Liu Hui initiates the computation by prescribing that a value for the diameter, 2 chi, be "set up" - a technical term referring to placing, on the surface for computing, a value on which the subsequent computations will be executed. As is common in Chinese mathematical writings, the whole text is formulated with respect to a given set of numerical data but it has a paradigmatic value: The numerical values mentioned hold for any other possible initial data. ${ }^{16}$

Note that what is "set up," right at the outset, comprises not only the initial numerical datum, but also its "meaning": it is the diameter of the circle. This feature will hold true for the whole text: the prescription of each operation or each sub-

\footnotetext{
${ }^{16}$ Evidence supporting this claim is given in Chemla 2003.
} 
procedure will be followed by a similar statement of its result. The value yielded by the operation or the sub-procedure, and the interpretation of the "meaning" of this result, will both be systematically given. Let me illustrate this point again by the next operation: halving the datum set-up. As we announced at the beginning of the section, the operation is prescribed directly, by means of a term naming the operation. ${ }^{17}$ The statement of the result can be decomposed into two parts: numerically, the operation yields $1 \mathrm{chi}$; and, semantically, halving the diameter will be interpreted as yielding the side of the hexagon. The dual nature of the result is essential for my argument. Thus, the text of the algorithm mentions the evolution of the values computed, while also progressively providing a geometrical interpretation of the result for each step. Therefore, finally, the "meaning" of the algorithm's result will be determined. The correctness of the procedure is established only if the meaning of the result corresponds to the magnitude sought. To designate the nature of the interpretation of the final result, Liu Hui uses a specific term: 意 ( $y i$ “meaning”). ${ }^{18}$ The term also refers to the successive interpretations of the meanings of the results of the preceding sequence of operations and sub-procedures composing the algorithm. Taken altogether, the "meanings" form the reasoning establishing the algorithm's correctness.

In the example, the second part of the result as formulated in the text ("the side of the 6-gon"), when taken from beginning till end of the algorithm, is precisely what constitutes Liu Hui's proof of his algorithm's correctness. I shall refer to the algorithms for which such proofs can be formulated as having a "transparent" structure. While reading the subsequent sentences of Liu Hui's text, I shall analyze the conditions required to make the sequence of interpretations possible. In the case of the previous operation of halving, formulating the second part of the result requires interpreting the result with respect to the figure. Let us observe how in the next part of the procedure, Liu Hui makes the meaning of the operations explicit:

"One takes half of the diameter, 1 chi, as hypotenuse, half of the side, 5 cun, as base (of the right-angled triangle), and one looks for the corresponding height. ${ }^{19}$ The square of the base, 25 cun, being subtracted from the square of the hypotenuse, there remains 75 cun. One divides this by extraction of the square $\operatorname{root}^{20}[\ldots$ description of the computation of anapproximation in the form of a sequence of units concluded by a decimal fraction, in the end

\footnotetext{
${ }^{17}$ This remark is important only because there are other modes of prescribing an operation that constitute another family of cases, in which the text of an algorithm refers to the reasons for its correctness (see below).

${ }^{18}$ I composed a glossary of technical expressions used in The Nine Chapters and its early commentaries (Chemla and Guo Shuchun 2004: 895-1042). In what follows, I shall refer to it as Glossary. It provides evidence for the meanings and facts regarding technical terms. For yi ("meaning, intention") see Glossary: 1018-1022.

${ }^{19}$ The terms I translate here by "base" and "height" are in fact technical terms referring, respectively, to the shorter and the longer sides of the right angle in a right-angled triangle.

${ }^{20}$ I follow the structure of the Chinese term for prescribing a square root extraction and underline, as the Chinese does, the link of that operation to division.
} 
simplified...]. Consequently, one obtains 8 cun 6 fen 6 li 2 miao 5 and three-fifths $h u$ for the height."

(令半徑一尺爲弦, 半面五寸爲句, 爲之求股。以句幕二十五寸減弦幕, 餘七十五

寸。開方除之, (...)。故得股八寸六分六嶅二秒五忽五分忽之二。; my emphases).

Two magnitudes, and their corresponding values, are now available: that of half of the diameter, which was computed, and that of the side of the hexagon, which was introduced as an interpretation of the result of that computation. Half of the side can thus be computed. Note that the computation of the latter value, along with its meaning, is prescribed indirectly by a mere reference to the result: "half of the side, 5 cun." (For other examples of indirectly prescribing operations essentially different from this one, see below). Even though the values of half of the diameter and the side of the hexagon are equal, their interpretations as segments differ, indicating the geometrical work required to formulate the interpretation of the operation of halving, as "side of the hexagon," not "half of the diameter." Moreover, the choice between these two possible interpretations (both to be used in the next step) is essential to allow the sequence of interpretations to, in the end, reach an adequate meaning for the result of the algorithm. By providing distinct geometrical interpretations of the same value, Liu Hui situates them as specific kinds of segments on the figure. Further, by granting to these segments the names of, respectively, "hypotenuse" and "base," he not only situates them with respect to each other on the diagram but also designates the right-angled triangle in which they play such parts (triangle OAB).

Chapter 9 of The Nine Chapters contains a problem, which, given the hypotenuse and base of a triangle, asks for the "height." The problem is followed, and solved, by a form of the "Pythagorean" procedure, the correctness of which Liu Hui discusses in that context. By using the term "looking for 求 qiu," in the text presently under examination, Liu Hui signals that he identifies the situation he is dealing with as similar to that of the problem in Chap. 9. He thereby justifies inserting in his algorithm, after the operations of halving, the procedure given in Chap. 9 for finding a triangle's height. This section of his algorithm reads: "21 "The square of the base, 25 cun, being subtracted from the square of the hypotenuse, there remains 75 cun. One divides this by extraction of the square root (...computation of an approximation...).. Consequently, one obtains 8 cun 6 fen 6 li 2 miao 5 and three-fifths $h u$ for the height" (emphasis mine).

This passage raises several issues related to our topic. First, note how the various operations are prescribed. As above, the squaring of the two known sides of the triangle is indicated by the statement of the result of the operation. By contrast, the terms by which the operations are prescribed (subtracting, dividing...) are common names for them.

Second, in contrast to the operation of "halving" discussed above, Liu Hui here prescribes the whole sub-procedure, of which only the final result is interpreted; there

\footnotetext{
${ }^{21}$ See the term “look for 求 qiu," in Glossary: 971. The corresponding problem and procedure in Chap.9 appear in Chemla and Guo Shuchun 2004: 704-707.
} 
is no need to interpret explicitly the meaning of the subtraction or other steps. Depending on the reasoning that is formulated in the interpretations of the successive results, either the result of an operation or that of a sub-procedure is provided; the operations of interpretation sometimes group together distinct computations into a single whole, when this is relevant for establishing the meaning of what is thereby computed.

Further, let us observe how the interpretation is achieved. The identification of a problem and the insertion of a procedure, the correctness of which was already established, allows Liu Hui to formulate the meaning of the result as "height" of the corresponding triangle and to situate it on the diagram (OA). Thus, both the problems and the procedures attached to them play parts in composing the algorithm and formulating the meaning of its sub-procedures. More generally, as the commentators bear witness, problems and their procedures play a key part in the two activities of composing, sub-procedure after sub-procedure, a desired algorithm and interpreting the sequence of results. This was probably already the case for the authors of the procedures in The Nine Chapters, which consists precisely of textual units composed by a problem and a procedure.

Last, note that at this stage, the two components of the result no longer have the same relation to the situation under investigation: the interpretation of the result as "height" is an exact meaning for the magnitude yielded, whereas the value is only an approximation. The two parts of the result run in parallel but no longer represent exact counterparts of each other.

In sum, the text for the algorithm as formulated by Liu Hui describes a sequence of operations (dividing, squaring, etc). For each operation, a value is yielded (exact or approximate), whereas the interpretation is provided for operations or blocks of operations.

The second part of the sequence of operations examined here can be interpreted in exactly the same terms. It reads as follows:

"One subtracts this (i.e., the height) from the half-diameter, 1 cun 3 fen 3 li 9 hao 7 miao 4 and three-fifths $h u$ remains, that one calls small base. Half of the polygon side then is called once again small height. One looks for the corresponding hypotenuse. Its square is 267949193445 $h u$, the remaining fraction being left out. One extracts the square root, which gives a side of the 12-gon."

(以減半徑, 餘一寸三分三嶅九毫七秒四忽五分忽之三, 謂之小句。触之半面而又

謂之小股。爲之求弦。其幕二千六百七十九億四千九百一十九萬三千四百四十五

忽, 餘分棄 之。開方除之, 即十二触之一面也。; emphases mine).

Some features of this part of the text with respect to the formulation of the algorithm and the meaning of its operations were not addressed in the discussion above. To begin, Liu Hui brings out the right-angled triangle $A B C$ by means of the same technique as above: He points out its base $\mathrm{AC}$ and its height $\mathrm{AB}$ by determining their values and indicating the part they play in the triangle. These two segments can be known on the basis of the magnitudes previously determined. The base is introduced as the meaning of an operation carried out on two segments known and placed in the diagram: half of the diameter and the height OA of the triangle OAB. As for its height, $A B$, introduced again as "half of the polygon side," it played another 
part in the triangle OAB. Reinterpreting the same segment in another way is required to formulate the meaning of the subsequent operations. So, Liu Hui restates the meaning of the segment, distinguishing triangle $\mathrm{ABC}$ from $\mathrm{OAB}$ by qualifying each of the sides of the former as "small."

Once the base and height of the triangle are determined, as above, by means of the term "one looks for" Liu Hui introduces the problem of finding the length of the hypotenuse. By contrast with the previous case, evoking the problem by way of its data and the desired result suffices here to indicate that the procedure - the "Pythagorean" procedure - is inserted in the algorithm composed. Indeed, even though the procedure is used for the computation of the square mentioned, it is not quoted in its entirety. Only its last two operations are listed explicitly. For the penultimate one, the approximation to be used for the numerical value it yields is given. As for the final one, note that Liu Hui makes only its meaning explicit - it is a "side of the 12-gon" - but not the value it yields. Clarifying why Liu Hui does this will allow us to understand a key characteristic of such algorithms, the structure of which I characterized above as "transparent."

\subsubsection{How Can the Structure of the Text for an Algorithm Lose its Transparency?}

To answer the question just raised, I examine the subsequent section of Liu Hui's text for his algorithm. It constitutes the beginning of the first repetition of the iterated sequence of operations:

"Procedure consisting in cutting the 12-gon in order to make a 24-gon: Likewise, one takes the half-diameter as hypotenuse, half of the side as base and one looks for the corresponding height. One sets up the square of the previous small hypotenuse, and one divides this by 4 , hence one obtains $66987298361 \mathrm{hu}$, and one leaves out the remaining parts, which gives the square of the base. This being subtracted from the square of the hypotenuse, what remains, one divides it by extraction of the square root $[\ldots]$

(割十二触以爲二十四触術曰: 亦令半徑爲弦, 半面爲句, 爲之求股。置上小弦

票, 四而一, 得六百六十九億八千七百二十九萬八千三百六十一忽, 餘分棄之, 即 句幕也。以減弦幕, 其餘, 開方除之, [‥]; emphasis mine).

The main idea of the procedure is the following: The previous computation had yielded the side of the 12-gon. Now, Liu Hui takes half of this magnitude, as before, as the base of a right-angled triangle, whereas half of the diameter is its hypotenuse. On this basis, the same procedure as before will yield this triangle's height. The procedure requires squaring the two data, subtracting the smaller from the larger, and extracting the square root. This algorithm can, as above, be interpreted either step by step or sub-procedure by sub-procedure to determine the meanings of the partial results. However, and this is a key point, that particular algorithm is not the one best suited for computations. As a result, Liu Hui will follow two distinct lists of operations, depending on whether he determines the meaning of the result or computes its value. In other words, the algorithm formulated to follow the meaning of 
the sequence of results differs from the algorithm followed for the computations. The reason is simple. At the end of the previous sequence of operations, Liu Hui had obtained the value of CB by extracting the square root of the value obtained, by means of a "Pythagorean procedure," for CB2. If we followed the operations just mentioned, we would extract a square root, divide that result by 2 and square the new result to enter it into the next "Pythagorean" procedure. Yet, in addition to the fact that the computations would be cumbersome, actually extracting the square root as Liu Hui does would increase the inaccuracy of the result. Instead of computing $[(\sqrt{ }(\mathrm{CB} 2)) / 2] 2$ - the sequence of operations he formulates to yield the result's meaning - Liu Hui uses another sequence of operations only for the computations; he obtains the value of $66987298361 h u$ by simply dividing CB2 by 4 . Thus, he introduces a distinction between the algorithm that shapes the meaning of the result and the algorithm that computes. The former can be represented by the formula $[(\sqrt{ } \mathrm{a}) / 2] 2$, whereas the latter boils down to [a/4]. This explains why only the meaning of $\sqrt{a}$, that is, $\sqrt{ }(\mathrm{CB} 2)$, not its value, needed to be determined: the operation is required for the algorithm determining the meaning of the result, not for the one that computes the value [a/4]. In fact, computing $[(\sqrt{ } \mathrm{a}) / 2] 2$ yields the same value as $[\mathrm{a} / 4]$ only if the result of a square root extraction is always given as exact. ${ }^{22}$ Yet the algorithm, as Liu Hui described it so far, does not give exact values for the results of root extractions. As a consequence, in terms of the "meaning" of the final result, there is no difference between the two sequences mentioned. However, as far as the values are concerned, the yielded approximations differ.

In sum, to go from the square of the hypotenuse corresponding to triangle $\mathrm{ABC}$ to the square of half of the side of the 12-gon, Liu Hui formulates two algorithms in parallel. The first extracts the square root, divides by 2 and then squares the value obtained; it corresponds to a text, the structure of which is transparent and the partial results of which can be interpreted directly, step by step, sub-procedure by subprocedure. This text is obtained by combining the reasons for using the operations, and thus its structure points to the reasons why the algorithm is correct. However, the algorithm is not convenient for the computations. It makes them uselessly cumbersome and increases their inaccuracy. Liu Hui thus follows a second algorithm for computing, one that rewrites the first algorithm's sequence of computations into one algebraically equivalent operation: "dividing by 4." Its starting point and end point are the same as the first algorithm's in terms of meaning. However, although it makes computation simpler, this rewriting causes a loss in the transparency of the text. There is a tension between the text that points out, by way of its structure, reasons for correctness and the text that prescribes more convenient computations.

The operations deleted in the latter need to be restored to retrieve a transparency similar to that of the first part of Liu Hui's text.

\footnotetext{
${ }^{22}$ Such transformations constitute parts of proofs to which I referred as "algebraic proofs in an algorithmic context." On this set of transformations and how their correctness was approached in ancient China, see Chemla 1997/1998.
} 
These simple remarks are yet fundamental: in most cases in which an algorithm's text is not structurally transparent with regard to the reasons for its correctness, one may infer that a similar rewriting occurred. That is, a list of operations carrying out a task, which was composed step by step, sub-procedure by sub-procedure, and whose structure was thus transparent, was rewritten so as to make the computations less clumsy. ${ }^{23}$ This conclusion casts light on how the transparency of the text for an algorithm can be achieved. It also explains why, in some cases, the commentators can interpret those texts for algorithms in The Nine Chapters that have a transparent structure, thereby making the reasons for their correctness explicit.

Here in our first example, we have read a section of the text large enough for us to draw some conclusions. With it, we could analyze one modality - the simplest one for writing down a text for an algorithm. Actions were prescribed in a straightforward way, by means of terms naming the operations to be executed. However, we also encountered some indirect ways of referring to actions: reference by stating the meaning of their results. Further, the text, or, more precisely, mainly the first part of the text, had a structure transparent about reasons for the algorithm's correctness. The meaning of the operations could be formulated, step by step, subprocedure by subprocedure, until the meaning of the result was established. In this text, Liu Hui formulated this meaning explicitly, combining the text that prescribes and the text that accounts for the correctness. The combination of the two became even more visible in the second portion, in which the two paths separated; that is, when, in order to compute a value for a magnitude, the list of operations leading to the meaning differed from that leading to a numerical value.

The part of the excerpt in which both dimensions coexist harmoniously can be considered a paradigm for such texts of algorithms in two ways. To bring these two ways to light, we shall consider separately the two components that the text combines.

To start with, texts for algorithms like the portion of the text in which operations are prescribed with transparent structure, in the technical sense I introduced above, frequently occur, not only in Chinese writings, like The Nine Chapters or the Book of Mathematical Procedures, ${ }^{24}$ but also in other mathematical traditions. Jens Høyrup's interpretation of Mesopotamian tablets recording texts for algorithm can be reformulated by saying that it implies that these texts have a transparent structure

\footnotetext{
${ }^{23}$ For those algorithms in The Nine Chapters the text of which does not have a transparent structure, the commentators regularly argue that the reason lies precisely in such rewriting. They compose, in the way just outlined, an algorithm carrying out the task expected from the algorithm commented upon. They further bring to light the cumbersome character of the algorithm they have composed, when it comes to computations, to account for the fact that the algorithm recorded in The Nine Chapters differs from the one they just composed. The transformations they describe in order to transform the latter algorithm into the former, thereby proving its correctness and accounting for its shape, constitute the part of the proof to which I refer by the expression of "algebraic proofs in an algorithmic context."

${ }^{24}$ See for example the texts for algorithms computing the volumes of solids recorded in bamboo slips 142-145 (Peng Hao (彭浩) 2001: 101-105). They share common features with texts for algorithms in The Nine Chapters and the structure of which the commentators interpret as transparent (Chemla 1991). Cullen 2004: 90-99 developed this idea of mine.
} 
(Høyrup 1990); thus, we have an entire corpus of tablets characterized by this feature. In addition, the texts for algorithms recorded in Al-Khwarizmi's Book of Algebra and al-Muqabala also share this property. ${ }^{25}$ The portion of Liu Hui's text examined is paradigmatic for all these sources.

However, the status of the "transparent structure" for texts is different in all these sources. This remark leads us to the second component of Liu Hui's excerpt, which makes explicit the meaning of the operations throughout the sequence which constitutes the text for the algorithm, thereby "interpreting" the structure of the text. In Liu Hui's excerpt and in al-Khwarizmi's book, the proofs of the correctness of the algorithms that the authors themselves developed share this feature: the proof follows the sequence of operations, as the text for the algorithm gives it, and makes explicit the meanings, step by step, or sub-procedure by sub-procedure. ${ }^{26}$ In this respect, the second component of Liu Hui's excerpt is paradigmatic. On the one hand, these sources all illustrate how the text for the algorithm is handled in writing down the proof of the correctness: the proof follows the text linearly, from beginning to end. ${ }^{27}$ On the other hand, we have testimonies that the structure of the text is meaningful for the authors who wrote it down. However, the evidence regarding the status of the structure is more indirect in the other cases. For The Nine Chapters, the structure can be showed to be meaningful for commentators, since the proof they write to establish the correctness relies on the structure of the text for the algorithm. With regard to the Book of Mathematical Procedures, by analogy with The Nine Chapters and its commentaries we can assume that the structure of the text was meaningful for readers. As for the Mesopotamian cases, except for similarities with Arabic sources in the formulation of algorithms that may indicate that we are justified to read the former in relation to the evidence provided by the latter, we could be left with no evidence regarding how readers made sense of the structure of the texts. However, these Mesopotamian texts have a second property that seems to also be aiming towards indicating reasons for correctness by means of the formulation of the algorithm's text. To understand this point better, I shall now turn to the second family of texts in The

\footnotetext{
${ }^{25}$ See the new critical edition and French translation in Rashed 2007: $100 \mathrm{ff}$.

${ }^{26}$ In the only case in al-Khwarizmi's book when the algorithm proved differs in its structure from the algorithm to be proved, we find two hints indicating that al-Khwarizmi's intention is to prove the algorithm with the structure with which its text is formulated. First, at the end of his proof, he addresses the differences between the two algorithms. Second, this is the only time when al- Khwarizmi develops a second proof, which in fact establishes the correctness of the algorithm, on the very basis of the structure of its formulation (see Rashed 2007: 108-113). Incidentally this remark shows that the structure of the text is not transparent in and of itself: It is made transparent by an interpretation.

${ }^{27}$ In both cases, the proof consists in making the meanings of the successive results explicit. However, the two authors carried out this operation differently. In the Liu Hui excerpt analyzed here, the meanings are made explicit in the text itself. However, al-Khwarizmi's book presents the proof as a separate text, the structure of which follows the structure of the text for the algorithm. Moreover, the dispositifs within which the meanings are expressed differ. Liu Hui makes use of diagrams as well as of problems and procedures attached to them. These are precisely the elements with which Liu Hui claims to have made the $y i$ (意, “meaning”) explicit (see yi in Glossary). Al-Khwarizmi uses only diagrams, the nature of which differs from Liu Hui's.
} 
Nine Chapters: Those texts that point out the reasons for correctness in how texts for algorithms are written, but use a different technique than we have previously discussed to indicate those reasons.

\subsubsection{A Necessary Digression: Aspects of Liu Hui's Practice of Proving the Correctness of Algorithms}

How texts belonging to the second family refer to reasons for the correctness of the algorithm is less easy to understand than the first family. Again, the commentators' testimony will prove essential to approach these texts in a rational way. In particular, as a necessary introduction, I shall briefly discuss the practice of proving the correctness of algorithms to which the commentaries on The Nine Chapters bear witness. An essential passage of Liu Hui's commentary in which he establishes the correctness of the procedure that The Nine Chapters provided to add fractions illustrates perfectly the features of proof needed for the argument. ${ }^{28}$ The procedure is formulated after three similar problems, of which the first asks:

(1.7) "Suppose that one has $1 / 3$ (i.e., one of three parts) and $2 / 5$ (i.e., two of five parts). One asks how much one gets if one gathers them."

(今有三分之一, 五分之二, 問合之得幾何。).

The procedure included by The Nine Chapters to solve such problems corresponds, in modern terms, to the formula $\frac{a}{b}+\frac{c}{d}=\frac{a d+c b}{b d}$. It can be used to add an arbitrarily large number of fractions. Its text reads:

"The denominators multiply the numerators that do not correspond to them; one adds up and takes this as the dividend. The denominators being multiplied by one another make the divisor. One divides $[\ldots]$."

(術曰：母互乘子, 扭以爲實。母相乘爲法。。實如法而一 $[\ldots]$ ).

The first sentence of the procedure, which prescribes a kind of multiplication ( $y$ hucheng $x$, "multiplying the $x$ 's by (each of) the $y$ 's that do not correspond to them"), translates into several operations on the surface for computing. In the case when the problem deals with two fractions, the sentence corresponds to multiplying $a$ by $d$ and $c$ by $b$. In a case of $n$ fractions, the sentence groups together all the multiplications of each numerator by all the other fractions' denominators. Thus, there is no one-to-one correspondence between the terms referring to operations in the text and the actions performed on the surface for computing. Moreover, the practitioner has to determine the relationship between the text and the actions on the basis of the problem to be

\footnotetext{
${ }^{28}$ I have devoted several publications to this text. I shall strictly limit myself here to what is essential to deal with the topic of this article. For greater detail, compare, for instance, Chemla 1997.
} 
solved. As the commentator will make clear, the sentence in question groups together operations that have the same "meaning."

In brief, Liu Hui approaches establishing the correctness of the procedure as follows: The expression for the fractions $\mathrm{m} / \mathrm{n}$ involved in the outline of a problem like 1.7, “ $m$ of $n$ parts" (n fen zhi m, $n$ 分之 $m$ ), gives the fractions as composed of "parts." I characterize this level as "material," as opposed to the "numerical" level, in which the stress is placed on the pair of numbers (numerator and denominator) defining the fraction. On the one hand, the statement of Problems like 1.7 gathers various disparate parts together to form a quantity that must be evaluated. On the other hand, the algorithm prescribes computations on numerators and denominators to form a dividend and a divisor. Establishing the correctness requires proving that the value obtained by division correctly measures the quantity formed by assembling the parts given.

In a first step, approaching the fractions as manipulated by the algorithm, Liu Hui stresses the variability of their expression: He underlines that one can multiply, or divide, both the numerator and the denominator by any given number without changing the quantity meant. In this particular context, to divide is to simplify the fraction. The opposite operation, to "complicate," which Liu Hui introduces in the context of his commentary on fractions, is needed only for the sake of the proofs. Liu Hui, then, considers the counterpart of these operations with respect to the fractions regarded as parts: Simplified fractions correspond to coarser parts, complex fractions to finer parts. The operation of "complicating" at the numerical level translates at the material level into disaggregating the parts. Again, at the material level Liu Hui stresses the invariability of the quantity, beyond possible changes in the way of composing it with parts.

Now, to prove that $\frac{a}{b}+\frac{c}{d}=\frac{a d+c b}{b d}$, Liu Hui shows that the strategy of the algorithm amounts to refining the disparate parts by "multiplication" so as to make them share the same size - in his words, "to make them communicate." This is the desired goal of the program when one considers the operation from the point of view of the fractions added, and Liu Hui has to connect this program to the operations prescribed. In order to uncover how the strategy is implemented, Liu Hui expounds the actual meaning of each step of the procedure in terms of both parts and numerators/ denominators, in order to make clear how the steps combined to fulfill the program announced. When "the denominators are multiplied by one another," an operation that in the course of the proof, he names "to equalize," this computes the denominator common to the fractions involved and defines a size that the different parts can share: they can thus be added. Moreover, when "the denominators multiply the numerators that do not correspond to them" to yield $a d$ and $c b$, the numerators are made homogeneous with the denominators to which they correspond; hence, the original quantities are not lost, Liu Hui says. Here too, he confers a name to this set of operations: "to homogenize." "Equalizing" the denominators and "homogenizing" the numerators, the algorithm thus yields a correct measure of the quantity formed by joining the various fractions. Thus, Liu Hui reasons, the procedure is correct. 
Liu Hui's new terms referring to the necessary operations do so in the same way as the term "multiplication of the $x$ 's by each of the $y$ 's that do not correspond to them" did: "Equalizing" corresponds to the action of multiplying, as many times as necessary, two or more denominators by one another, depending on the number of fractions dealt with. Moreover, "homogenizing" comprises in a single term all the multiplications needed to compute numerators homogeneous with the newly formed denominator. The key point for us here is to observe how the terms introduced in the proof refer to the actions to be carried out. "Equalizing" and "homogenizing" do not prescribe these multiplications directly. Instead, they refer to the actions to be taken by way of the "meaning" that the operations have in their context of use (in the sense of the word "meaning" introduced in II.2, above). In other words, the operations are prescribed by means of terms designating the intention that commands their use: one multiplies denominators so as to yield an "equal" denominator and thereby determine an "equal" size for the "parts" of the fractions involved. The same principle holds true for "homogenizing." The terms "equalizing" and "homogenizing" thus each designate groups of multiplications that achieve one and the same goal. In addition, Liu Hui introduces the operation "making communicate" as a step of the proof, capturing an overarching meaning in the main part of the procedure: It brings into "communication" parts that were disparate, allowing them to be added. However, the term corresponds to no specific step in the procedure, being in fact decomposed into and specified by the operations of "equalizing" and "homogenizing." The name of the overall strategy discloses the key goal of using the latter two operations: "equalizing" and "homogenizing" conjoin in making the parts share the same size and hence enabling them to "communicate."

Liu Hui perceives the operations "equalizing" and "homogenizing" as an alternative way of writing a text for an algorithm corresponding to the same set of actions on the surface for computing. This observation derives from the fact that in some contexts, he actually uses them, as later mathematicians like Zhu Shijie would also do, to prescribe how to add up fractions. However, the two ways of writing down a text for the same course of actions do not seem equivalent in his eyes, judging by the final remarks he makes regarding the operations introduced, for instance: "[...] If so, the procedure of homogenizing and equalizing is essential. [...] Multiply to disaggregate them, simplify to assemble them, homogenize and equalize to make them communicate, how could those not be the key-points of computations/ mathematics?"

I have argued elsewhere that these remarks can be interpreted as underlining that the terms "equalizing" and "homogenizing" have a second meaning, both in this context and in the other contexts in which they occur conjointly in the commentaries. For instance, in addition to its meaning in relation to fractions (equalizing denominators at the numerical level as well as equalizing the size of the parts at the material level), the term "equalizing" takes on a formal meaning. In each of the contexts in which Liu Hui discloses the pattern of equalizing and homogenizing, the terms highlight that the algorithm under consideration formally proceeds through 
making some quantities equal and making other quantities that are linked to them by a linear relation homogeneous of them. ${ }^{29}$ The expression of this second meaning is one key reason for which the two texts corresponding to the same actions are not equivalent. To conclude, in establishing the correctness of The Nine Chapters' procedure to add up fractions, Liu Hui pursues two goals simultaneously. On the one hand, he makes the "meaning" of the operations clear with respect to fractions: Their parts are disaggregated in concordant ways. On the other hand, he does so in such a way as to bring to light a "pattern," a "form," in how the material operations are carried out: They equalize and homogenize. This form discloses similarities between apparently unrelated algorithms. This description of Liu Hui's way of proving the correctness of the algorithm for adding fractions also accounts for his practice in other contexts in which equalizing and homogenizing occur. Although in each context they may have different concrete meanings, the fact that Liu Hui manifests the same pattern of proceeding in various contexts brings to light a formal strategy common to otherwise distinct algorithms. In addition, our reading of the proof Liu Hui developed in this piece of commentary shows how he produced a new text that prescribed an algorithm by stating the meaning of its operations: that is, the reason for using them. The Nine Chapters contains texts for algorithms precisely of this type. I shall now examine one of them, once again relying on Liu Hui's commentary on it.

\subsubsection{Texts for Algorithms Covering Various Cases and Referring to Operations by Way of their Meaning}

I shall illustrate the second family of texts with the example of the algorithm given in The Nine Chapters to divide quantities combining integers and fractions. ${ }^{30}$ The text is placed after two problems, which read:

(1.17) "Suppose one has 7 persons sharing 8 units of cash, $1 / 3$ of a unit of cash. One asks how much a person gets."

(今有七人, 分八錢三分錢之一。問人得幾何).

\footnotetext{
${ }^{29}$ To give but one example, Chap. 8 in The Nine Chapters is devoted to solving systems of linear equations. The algorithm provided for this is the so-called "Gauss elimination method." In his account for the correctness of this procedure, Liu Hui brings to light that it "equalizes" the coefficients of the unknown that is eliminated, whereas it "homogenizes" the other coefficients in the equations between which one eliminates. At a material level, the operations of equalizing and homogenizing have a meaning that differs from those occurring in relation to fractions. However, at a formal level, the algorithms share the same strategy.

${ }^{30}$ I argued for an interpretation of this text in Chemla 1992. In a forthcoming paper, I examine how the text covers the various cases in greater detail. This paper will be published in the volume edited by $\mathrm{J}$. Virbel and myself, as the outcome of the seminar "History of science, history of text." Here, I rely on my 1992 publication without repeating its argument, my main focus being to analyze the text of the algorithm from the perspective of how it refers to reasons for correctness.
} 
(1.18) "Suppose again one has 3 persons and $1 / 3$ of a person sharing 6 units of cash, $1 / 3$ and $3 / 4$ of a unit of cash. One asks how much a person gets."

(又有三人三分人之一, 分六錢三分錢之一、四分錢之三。問人得幾何).

The problems are followed by a text for a procedure, however, at first sight, the meaning of this text is obscure for a present-day reader. I translated it in such a way as to keep the flavor of the original text, as follows:

"One takes the quantity of persons as divisor, the quantity of cash as dividend and one divides the dividend by the divisor. If there is one type of part, one makes them communicate. [here, Liu Hui inserts a commentary on the algorithm] If there are several types of parts, one equalizes them and hence makes them communicate."

(以人數爲法, 錢數爲實, 實如法而一。有分者通之； 重有分者同而通之。； emphases mine).

In the Chinese text, as in the English translation, the terms I marked in bold prescribe operations indirectly, in contrast with the straightforward way of referring to operations in the previous examples of texts for algorithms in The Nine Chapters. Since we are not members of the scholarly culture for whom these indirect prescriptions made sense, we are not in a position to understand them and translate them into action, let alone analyze them. However, we are able to perceive that this mode of prescribing operations does relate to the type of proof described in the previous section. Fortunately, we can rely on Liu Hui - the most ancient reader available to us to observe - to determine for us, through his eyes, the actions corresponding to the text. I shall examine his interpretation, before analyzing his view of how these indirect speech acts - or, in this case, "indirect scribal acts" - are carried out.

Liu Hui interprets the text as dealing with several cases. The first and most fundamental case corresponds to no actual problem in The Nine Chapters: it is the case in which the two data are integers. The algorithm then boils down to its first part, directly prescribing a division.

The case in which the data contains only one type of fraction occurring in the dividend and/or the divisor, partly illustrated by problem 1.17 , is dealt with by a sequence of actions that can be represented, in modern terms, by the following formulas: ${ }^{31}$

$$
\begin{gathered}
\left(a+\frac{b}{c}\right) / d=(a c+b) / d c \\
\left(a+\frac{b}{c}\right) /\left(d+\frac{e}{c}\right)=(a c+b) /(d c+e)
\end{gathered}
$$

\footnotetext{
${ }^{31}$ In fact, the general case meant here corresponds to the second formula, the first corresponding to $e$ equal to 0 .
} 
These computations, as Liu Hui explains, translate into action the prescription "one makes them communicate." This operation, which constitutes the second section of the text, transforms $\left(a+\frac{b}{c}\right)$ and $d$ (or $\left(d+\frac{e}{c}\right)$ ) into, respectively, $(a c+b)$ and $d c$ (or $(d c+e))$, that is, into a problem in which we recognize the fundamental case. The data characteristic of the third and final case - where two (or more) different fractions are involved, as illustrated by problem 1.18 - are transformed, by the operation of "equalizing," into what can be represented as follows:

$$
\left(a+\frac{b}{c}\right) /\left(d+\frac{e}{c}\right)=\left(a+\frac{b f}{c f}\right) /\left(d+\frac{e c}{c f}\right)
$$

Clearly, the operation of "equalizing" transforms the problem back to the second case. This interpretation fits with the fact that the next operation prescribed in this segment of the text is to "make them communicate," which returns them to the fundamental case. In brief, the text for the algorithm presents the various sets of actions to execute a division, sorting them out into three cases of increasing complexity. The actions necessary for solving problems falling under the last case embed those required for the second case. Both sequences embed the operations solving the fundamental case, which constitute in a sense the root of the text. ${ }^{32}$

Liu Hui's commentary here contains two layers. In one, he translates the indirect prescriptions into terms that prescribe the operations straightforwardly. In the second, exactly in the same way as for the addition of fractions, he elucidates that the terms "equalizing" and "making communicate," used this time in the text itself, indicate the "meaning" of the actions to be performed; in other words, the reasons why these actions conjoin into a correct algorithm. This testimony proves that Liu Hui interprets the indirect speech acts as prescribing the computations by stating the reasons why they should be carried out. Thus, in Liu Hui's view, the text for the algorithm recorded in The Nine Chapters refers to reasons for its correctness.

The text just examined achieves that property in a way that contrasts sharply with that I described above in Sect. 17.3.1. In the earlier example, the text presented the algorithm in the form of a sequence of operations, the structure of which was transparent; that is, the "meaning" of which could be formulated step by step, or subprocedure by sub-procedure. Liu Hui, when meeting such texts, makes explicit the "meanings" thereby indicated. The second type of text, illustrated by the last example, designates the reasons for correctness by means of the terms chosen to prescribe the operations: These operations are prescribed indirectly by the reasons for using them. Again, Liu Hui develops proofs that make these meanings explicit. The feature of indirectness characterizes texts that belong to the second family, whereas transparency

\footnotetext{
${ }^{32}$ My forthcoming article points out that such types of text, organizing cases in exactly the same way, recur in Chinese sources from the second century B.C.E. till at least the seventh century. The next section of this article will show another example of this phenomenon. The way in which the practitioner used the text to derive lists of actions requires clarification. It illustrates how, behind what appears to be a list of operations, complex structures may be hidden. However, I cannot dwell on this issue here.
} 
captures the essence of the first family. I indicated above that Høyrup's analysis of Mesopotamian texts implied that they belonged to the first family. However, things seem to be subtler in this case: Høyrup not only shows that the structure of the text allows us - and probably also the practitioners - to interpret the meaning of the operations geometrically in a progressive way, but also suggests that the terms used to prescribe the operations simultaneously indicate the geometrical operation to be carried out to account for the whole procedure's correctness. In other terms, we may cautiously assume, given that we have no testimony of how ancient readers interpreted these texts, that the Mesopotamian texts in Høyrup's analysis belong simultaneously to both families. They use both of the two main techniques illustrated here in order to indicate, by way of the text of the algorithm itself, reasons for its correctness. Thus, by making use of the distinction introduced here, the historian can disclose various ways in which practitioners used different possibilities for writing texts for algorithms.

However, going one step further in this analysis will yield further source material for historians. In fact, different Chinese sources bear witness to two distinct ways of realizing texts from the second family identified. More precisely, the way in which the property characterizing the second family of texts is implemented in The Nine Chapters is specific. The way in which the terms state the reasons is coherent with Liu Hui's commentary on the addition of fractions: the terms indicate the reasons, while disclosing simultaneously a "form" in the computations. This last feature is essential for the new distinction just formulated, because the way in which the reasons are indicated by the terms chosen to prescribe the operations differs in other contexts from that in The Nine Chapters (e.g., as I shall show in the next section, in the Book of Mathematical Procedures).

In order to prepare the description of this contrast, I shall examine in greater detail how Liu Hui interprets, in the text for the division analyzed above, the term "one makes them communicate." This implies returning to the second case, which deals with divisions such as: $\left(a+\frac{b}{c}\right) /\left(d+\frac{e}{c}\right)$. As we saw, Liu Hui translates the prescription in question into the two sequences of actions that lead to computing, respectively, $(a c+b)$ and $(d c+e)$. But how does Liu Hui understand that these actions are prescribed by the term "one makes them communicate"?

Lui Hui relates the use of the term to two main facts. First, computing $a c$ and $d c$ consists in carrying out a multiplication that, on a material level, disaggregates the integers $a$ and $d$, "making" the integers "communicate" with the numerator. One can thus add them, which yields $(a c+b)$ and $(d c+e)$. The operation is prescribed by neither the term, "one multiplies," nor by the term that would capture the reason at a material level, "one disaggregates." Rather, the operation is prescribed by the reason expressed in a way that highlights a general "form" in the computations. At that level, the use of "making communicate" echoes how other algorithms, like that to add up fractions, proceed, even though the specific operations meant are different. Moreover, the use of "making communicate" falls under the rhetorical category of the synecdoche: as Liu Hui understands it, designating the reasons for carrying out the multiplications also prescribes the ensuing additions $(a c+b$ and $d c+e)$. 
However, the use of "making communicate" also captures another feature in the procedure - this is the second fact that Liu Hui associates with it. This second feature corresponds to no specific action but is essential for the computations to be correct. These computations bear on what will eventually be a dividend and a divisor. The data are thereby "brought into relation" by the fact of eventually being terms of a division. As a consequence of "being in relation," they "are made to communicate," a second layer of meaning that the term here conveys in Liu Hui's eyes. This implies, Liu Hui stresses, that their values must be modified simultaneously - multiplied or divided by the same number - in order for the result of the division not to be changed. In fact, Liu Hui approaches this property of quantities being brought into relation in the most general way possible, indicating that these phenomena are general and that sets of quantities sharing such properties fall under the rubric of the general concept of liu, which he introduces on that occasion. ${ }^{33}$ Observing the computations carried out from this perspective, one notices that the algorithm proceeded in such a way that it transformed the would-be dividend and divisor simultaneously and in the same way, multiplying both by $c$. The fact that the quantities in question "are made to communicate," by being made terms of a division warrants the correctness of the set of multiplications with respect to the outcome of the final division. In the end, this property warrants that the second case can be reduced to the first one. Hence, this aspect of "making communicate," which Liu Hui brings to light, corresponds to no action but discloses another reason, linked to the "communication" between values, that accounts for the algorithm's correctness.

To recapitulate, the term "making communicate," as Liu Hui comments on it, designates a set of elementary actions and properties (the main property being that the data that become "dividend" and divisor" "are made to communicate"). The term refers to a cluster of operations and properties in relation to the fact that they receive the same "meaning" and hence are shown to be correct as a whole. In other words, the cluster has a "meaning" and the procedure refers to it by way of this "meaning." This analysis shows how a term in the text of an algorithm can both prescribe a set of actions and correlatively convey a conceptualization of the transformations carried out. The grain of the initial description here was particularly coarse and, in relation to that, loaded with meanings that Liu Hui unpacks. Comparing Liu Hui's uses of "making communicate" in this context and in his proof of the correctness of the algorithm to add fractions enables an even finer interpretation: even though formally in each context the actions meant by the term allow the data to enter jointly into certain common operations, the actual computations required to do so differ in each context.

The use of these types of terms and operations characterizes The Nine Chapters and its commentaries. This fact emerges from a comparison with the texts for algorithms in the Book of Mathematical Procedures, to which I now turn.

\footnotetext{
${ }^{33} L \ddot{u}$ qualifies quantities that are defined only relatively to each other - see below. This concept was discussed in Li Jimin (李繼閔 1982) and in Guo Shuchun (郭書春 1984). See also Glossary, 956-959.
} 


\subsection{Relationships Between Texts for Algorithms and Reasons in the Book of Mathematical Procedures}

The Book of Mathematical Procedures also contains texts for algorithms of the first family, essentially similar to those included in The Nine Chapters some two centuries later. However, I shall focus on its texts that make use of techniques specific of the second family, concentrating, in particular, on how they are formulated.

I shall examine closely the text for an algorithm that executes an operation called in the Book of Mathematical Procedures "lü-ing with the dan." The dan (石) designates a unit of measure. ${ }^{34}$ If we rely on the occurrences of the expression "lü-ing with the $d a n$ " in the book, we see that the operation computes the price for 1 dan of something, given the price for another quantity of the same thing. The character lï used here is the same as the one Liu Hui later used in his commentary on The Nine Chapters' algorithm for division above. Although, Liu Hui mostly used the term lü as a noun, the Book of Mathematical Procedures and the related sections in The Nine Chapters itself used it mostly as a verb. I have shown elsewhere (Chemla 2006) that, when recording exactly the same procedures to carry out operations having names of the kind "lü-ing with the dan," The Nine Chapters renamed two quantities involved in the Book of Mathematical Procedures' computations with the character lï. This fact seems to indicate a historical connection between these algorithms and the emergence of the concept of lï. In addition, it suggests that the interpretation of lï in the Book of Mathematical Procedures should, at least as a first hypothesis, rely on this later development. Hence, I here interpret $l \ddot{u}$ as referring to the fact that the algorithm will choose " 1 dan" as making a set of lü with the quantity of something given in the statement of the problem to be solved, in the sense outlined in the preceding section. I shall however, at least for the moment, leave lü untranslated.

I shall first examine a problem for which the operation is executed and the algorithm described in a straightforward way before turning to the text provided for its more general statement. The problem recorded in bamboo slip 76 reads as follows:

\footnotetext{
${ }^{34}$ I am grateful to Professor Ma Biao, who has established that the reading of the character 石, when it designates a unit of measure for capacities, should be dan, and not shi as occurs in most Western sinological literature. I refer the reader to his forthcoming article on the topic. When the Book of Mathematical Procedures was composed, this character designated both the highest unit of capacity and the highest unit of weight used. In both cases, it read dan. There are reasons to believe that both units of measures are meant in the title of this operation and that they paradigmatically refer to the highest unit in a given series of units. The critical edition of the part of the Book of Mathematical Procedures that I analyze here can be found in Peng Hao (彭浩 2001: 73-75). Note that the manuscript found in a tomb was written on bamboo slips, which were discovered unbound. In such cases, the operations of the critical edition include suggesting an order of the bamboo slips. The order for the slips to which I refer is the one suggested by Professor Peng Hao. Below, we shall refer to two series of units. For the units of weight, the relationships between them are given in slip 47, as follows: 24 zhu for 1 liang, 384 zhu for 1 jin, (...), 46080 zhu for 1 dan. We can deduce the relationships between the units of capacity used in the Book of Mathematical Procedures from its text. They are, respectively, 10 sheng for $1 \mathrm{dou}, 100$ sheng for $1 \mathrm{dan}$. These values correspond to what contemporary sources attest to.
} 
"Trading salt Suppose one has 1 dan 4 dou 5 sheng 1/3 sheng salt and that when trading it, one obtains 150 cash. If one wants that the dan "lü's" it (the quantity of salt bought), how much cash does this make? One says: 103 cash 9[2]/43[6] cash."

(賈鹽 今有鹽一石 四斗五升少半升, 賈取錢百五十, 欲石a（率）之, 為錢幾何」。 日: 百三錢四百 卅(三十)[六]分錢九十[三]。/76/).

In other words, for a given amount of cash, one trades an amount of salt, which is expressed with several units of capacity and a fraction. The question is: how much cash corresponds to a given unit of capacity, here the dan? The idea put into play in the algorithms for solving this category of problems, whether in The Nine Chapters or in the Book of Mathematical Procedures, is to apply a rule of three. In modern terms, the algorithm can be represented by the formula:

$$
\frac{\text { cashmultiplied by 1unit(dan) }}{\text { quantity bought }}
$$

According to the way in which the rule of three was handled in ancient China, the divisor and one term of the product that makes the dividend are considered as lï. The algorithm first transforms, simultaneously and in the same way, the unit (1 dan) and the quantity bought - that is the two "lü's," the first in the dividend and the second in the divisor-, so as to turn them into integers. Only then are the operations multiplication and division - executed. The end point of these transformations can be represented by the following formula:

cashmultiplied by1unit(dan) expressed inthe sameunit as the divisor

quantity bought expressed with respect to a unit inwhichthe quantity becomes aninteger

As for the sequence of transformations, it amounts to the following operations:

$$
\begin{gathered}
\frac{\text { cashmultiplied by } 1 \text { unit }(\text { dan })}{\text { quantity bought }}=\frac{\text { cashmultiplied by } 1 \text { unit } u 1}{q 1 u 1+q 2 u 2+\frac{m}{n} u 2} \\
=\frac{\text { cashmultiplied by } n .1 \text { unit } u 1}{n q 1 u 1+n q 2 u 2+m u 2}
\end{gathered}
$$

and if $u 1=k 1 u 2$

$$
=\frac{\text { cashmultiplied by } n \cdot k 1 u 2}{n q 1 k 1 u 2+n q 2 u 2+m u 2}
$$

This sequence of transformations is described in the text of the algorithm associated with this particular problem as follows:

"Procedure: One triples the quantity of salt, which is taken as divisor. One also triples the quantity of sheng of 1 dan, and with the cash, one multiplies it, which is taken as dividend."

(术 $($ 術)/76/曰：三鹽之數以為法, 亦三一石之升數, 以錢乘之為實。/77/; emphasis mine).

The procedure stated is specific to the stated problem, using its data. It refers to operations straightforwardly and as a sequence of prescriptions to be followed. 
However, it has a "shape": the way the transformation of 1 dan is expressed underlines, with the use of the word "also," that it is parallel to the transformation undergone by "the quantity of salt." This "also" would be useless if the text was a pure sequence of prescriptions. One might suggest that this way of emphasizing a structure in a sequence of operations points to the operations' meaning - where the meanings can be made explicit step by step - which would make the text a part of the first family.

However, much more interesting for us, is the text provided in the same book for the general algorithm, which Peng Hao chose to place right before the specific problem and procedure just mentioned. This general text does not seem to be associated with any specific problem. I shall translate it to give a flavor of its formulation. Again, its interpretation requires that the reader be trained in the scholarly culture in which the text was composed. I shall then offer an interpretation for it within the framework of the example of the previous problem. The text reads:

"lü-ing with the dan Procedure for lü-ing with the dan: One takes what is exchanged as divisor. One multiplies, by the cash obtained, the quantity of 1 dan, which is taken as dividend. Those for which, in their lower (rows), there is a half, one doubles them; (those for which there is) a third, one triples them. Those for which there are dou and sheng, jin, liang and zhu, one also breaks up all their upper (rows), one makes the (rows) below join them, (yielding a result) which is taken as divisor. What the cash was multiplying is also broken up like this."

(石a（率）石 $\mathrm{a}$ (率)之術曰：以所買=（賣）為法, 以得錢乘一石數以為實。其下 有半者倍之, 少半者三之, 有斗、升、斤、兩、朱（銖）者亦皆//破其上, 令下從 之為法。錢所乘亦破如此。/74-75/; my emphases)

The interpretation of the text that I suggest relies, not only on the problem quoted above, but also on hypotheses regarding the use of the surface of computing to which the Book of Mathematical Procedures refers (see Figs. 17.2-17.5 below). ${ }^{35}$ Step by step:

1. "One takes what is exchanged as divisor. One multiplies, by the cash obtained, the quantity of 1 dan, which is taken as dividend."

(以所買 $=$ (賣) 為法, 以得錢乘一石數以為實。).

The terms of dividend and divisor refer to, respectively, the middle and the lower rows of the surface. When the division is executed, the quotient is progressively placed in the higher row. In the case of the procedure analyzed, what is placed in the middle row is the setup of a multiplication. Each row can become the space in which an operation can be set up. Here the multiplicand and multiplier are placed in subrows of the middle row, according to the usual setup of a multiplication: the multiplier is in the higher sub-row, the multiplicand in the lower one. However, although the terms of the operations are set up, neither the division nor the multiplication seem to

\footnotetext{
${ }^{35}$ To support my reconstruction of the use of the surface for computing, see my description in Chemla and Guo Shuchun 2004. Simply, I use Arabic figures in place of the configurations of counting rods with which in ancient China figures were written down on the surface. Moreover, for a more detailed discussion of the interpretation provided, see Chemla 2006.
} 
be executed at this point, since several terms will undergo transformations before the main operations are carried out (see below). Exactly the same thing occurred in the sequence of transformations of formulas above: it presented multiplications and divisions, and modified their terms before they were executed. This phenomenon also appears in the text of the algorithm for division examined above. Last, the quantity placed in the position for the divisor comprises several units and a fraction. In my interpretation, the lower unit associated with an integer is placed in the middle subrow of the lower position, whereas the larger units are placed in the sub-rows above it, and the fractions horizontally (numerator on the left, denominator on the right) in the sub-rows under it. The initial configuration thus resembles Fig. 17.2.

2. "Those for which, in their lower (rows), there is a half, one doubles them; those for which there is a third, one triples them."

(其下有半者倍之, 少半者三之; my emphases)

The text now turns to examining cases in which the quantity exchanged includes fractions. Later, it prescribes what to do in cases where the quantity contains more than one unit from a series. In other words, the text encompasses several types of cases and gives sequences of actions to be followed depending on the particular case encountered.

In case there are fractions, one has to multiply the quantity in the divisor position (i.e., each of the rows constituting it), by the denominators of these fractions. This operation is prescribed in a new indirect way; that is, by a simple enumeration of two paradigmatic cases and the specific action that they require. A similar kind of prescription will be chosen in the next sentences. If there is no fraction, the practitioner skips this sentence when deriving actions from the text. However, the sentence must, in any case, be read. For our example, the sentence prescribes actions that lead to the configuration in Fig. 17.3.

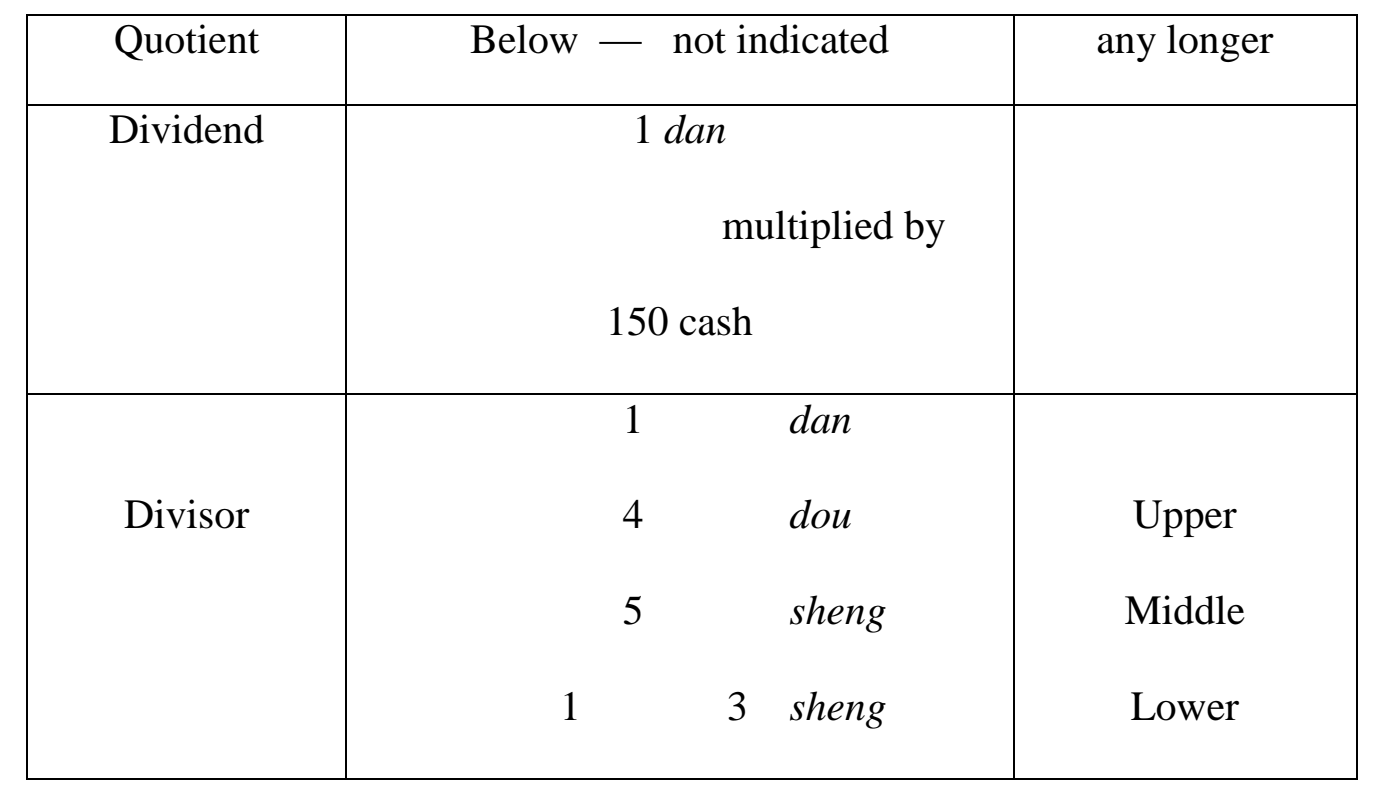

Fig. 17.2 The first step in the use of the surface of computation 


\begin{tabular}{|l|cc|c|}
\hline Dividend & 1 dan $r$ & \\
& \multicolumn{2}{|c|}{ multiplied by } & \\
\hline Divisor & 3 & dan & \\
& 12 & dou & Upper \\
& 15 & sheng & Middle \\
& 1 & (3) sheng & Lower \\
\hline
\end{tabular}

Fig. 17.3 The second step in the use of the surface of computation

The next step contains the key phenomenon of interest here:

3. "Those for which there are dou and sheng, jin, liang and $z h u$, one also breaks up all their upper (rows)."

(有斗、升、斤、兩、朱（銖）者亦皆//破其上; my emphasis)

As above, the general possibility that there be more than one unit in the quantity exchanged is expressed by an enumeration of two specific cases. Each of these cases is itself formulated as an enumeration: The quantity would have either two units from the series of units of capacity or three units from that of weight, both enumerations listing units smaller than the dan, which both series have as their largest unit.

The main feature of interest here is the prescription with the expression "one also breaks up...." That the text underlines "also" implies that the operation meant is a multiplication, as in Sentence 2 above. This explains my assumption that, even if there is no fraction and Sentence 2 is irrelevant with respect to the actions carried out, the practitioner using the text must read Sentence 2 for the "also" in Sentence 3 to make sense. As in the procedure for the specific problem on bamboo slip 76 examined above, the "also" would be of no use if the text were merely prescriptive.

What needs to be multiplied is made clear: the operation is to be executed on "all the upper (rows)" (皆...其上) in the quantity placed in the position of the divisor, that is, "all the rows" above the middle one, in which the smaller unit is placed. This leads to the configuration in Fig. 17.4.

But the essential issue is how the multiplications are designated. The term "break up" indicates the actions indirectly. This indirect speech act designates the multiplications by the intention for using them: to break up all the higher units so as to convert them into the smaller unit appearing on the surface. The text thus simultaneously uses different ways of prescribing operations. Two remarks are interesting at this point. 


\begin{tabular}{|c|c|c|}
\hline Dividend & $\begin{array}{r}\text { multiplied by } \\
\\
\text { Divisor }\end{array}$ & \\
& 150 cash & \\
& 120 & Upper \\
& 15 & Middle \\
& 1 & Lower \\
\hline
\end{tabular}

Fig. 17.4 The third step in the use of the surface of computation

First, the term "break up" evokes the term "disaggregating" that Liu Hui repeatedly uses in his commentary on fractions from The Nine Chapters. There is a continuity between the terms by means of which the Book of Mathematical Procedures refers to multiplications in this context and the reasons as formulated by Liu Hui in a similar context. This connection supports my interpretation that in the present case the operation of multiplication is prescribed by way of the reason to make use of it.

Second the "also" in Sentence 3 makes the meanings circulate both ways. It not only supports the interpretation of the prescription "to break up" as referring to a multiplication but also retrospectively transmits the meaning "breaking up" to the multiplications prescribed by Sentence 2. Here too, such a meaning is continuous with how Liu Hui would use it in his commentary on The Nine Chapters. Most important, however, "break up" refers to multiplication by stating its "material" meaning, not by capturing its meaning in any formal way. This constitutes the key difference between The Nine Chapters and the Book of Mathematical Procedures: When prescribing operations by stating the reasons for using them, the former book uses reasons formulated so as to capture a general form in the computations, whereas the latter uses reasons formulated at a material level.

Sentence 4 simply prescribes adding up all the rows in the divisor position, which by this point have all been converted into the same unit. It reads:

4. "One makes the (rows) below join them, (yielding a result) which is taken as divisor." 36 (令下從之為法。).

\footnotetext{
${ }^{36}$ Note that the same term "divisor" designates different values at different points in the flow of computations. This is one of the many examples of the use of the "assignment of variables" in ancient Chinese texts of algorithms.
} 
The fifth and final sentence again presents the phenomenon in which we are interested in a way that allows further conclusions:

5. "What the cash was multiplying is also broken up like this."

(錢所乘亦破如此; my emphasis).

I shall discuss the interpretation of this sentence piece by piece. "What the cash was multiplying" designates the "1 dan" by the operation involving it in Sentence 1. However, this operation, by means of which the value " 1 dan" is indicated, was not executed then, since one of its terms is now to be modified. ${ }^{37}$

Further, for the second time in this text an "also" occurs. Here too, it indicates that two parallel procedures are used in the sequence of actions. However, what is designated here, as well as how it is designated, is different. Now the procedure reused is the one that modified the quantity in the divisor, and it is signified as "like this." So the list of actions meant by this "also" depends on the case to which the procedure is applied. The prescription simply indicates that the procedure to be applied to 1 dan is the same one needed to apply to the quantity in the divisor, depending on its fractions and list of units. In our example, the procedure involves multiplying by 3 and transforming into sheng. It yields the configuration in Fig. 17.5.

Note how this procedure is designated again by the verb "break up": Understanding this text demands that the transformation linked to the presence of fractions, upstream, be understood as "breaking up." Only in such a case can the appropriate series of actions be understood as "breaking up in the same way," again, a quite coarse-grained description. Moreover, the series of actions is indicated by the reasons that make the operations necessary; that is, by the intention of the set of actions. But in prescribing actions a second time with the same term, the author of the text is confident that the reader will know how to translate the same reason into different actions; that is, the different actions will be determined by when, in the flow of computations, the reason must be fulfilled.

Finally, as in the previous case and in contrast to The Nine Chapters when it designates actions by their reasons, the text in the Book of Mathematical Procedures designates actions by their material meaning, not their formal one. Nevertheless, the text analyzed here still prescribes actions indirectly by means of the reasons for carrying them out. Consequently, the text itself also formulates reasons for the correctness of the algorithms. This text, thus, also belongs to the second family of texts that I identified.

Still, this last example raises the questions of the reason for such a difference between the Book of Mathematical Procedures and The Nine Chapters, as well as its bearing on the issue of the historical connection between the two writings.

\footnotetext{
${ }^{37}$ The 1 by which the amount of cash was supposed to be multiplied will now be modified. This explains why I initially suggested not executing the multiplication immediately. This recalls how the text for division is formulated in The Nine Chapters.
} 


\begin{tabular}{|l|c|c|}
\hline Dividend & 300 & \\
& multiplied by & \\
& 150 cash & \\
\hline Divisor & 436 & \\
& & \\
\hline
\end{tabular}

Fig. 17.5 The fourth step in the use of the surface of computation

\subsection{Conclusion: Writing Texts for Algorithms and Understanding}

These analyses clarify how anachronistic and naïve an approach to texts of algorithms can be, especially one that holds that these texts refer to operations only by name, and boil down to a sequence of computations to be executed in the order in which the terms prescribing them occur. Such is not the case in ancient texts. In the examples I examined, the relationship between the text for an algorithm and the actions carried out on an instrument is by no means straightforward. For example, the last text examined showed the case of a multiplication that was prescribed initially but not executed until later. In addition, in the same text, the order in which the operations were to be executed was far from obvious. In the text for division, the way in which cases are covered by a single text differs from expectation. Last, in several cases elementary actions were grouped under a single term, the meaning of which was not always straightforward - sometimes, this feature related to the indirect reference by the text of an algorithm to actions by giving the reasons for carrying them out.

These observations recall the issue of proof. The detailed descriptions here disclosed two main ways in which the text for an algorithm can indicate reasons why the algorithm is correct.

First, some texts for algorithms are written in such a way that the structure of the list of operations constituting them is "transparent." In other words, the meaning, or intention, of the operations or blocks of operations can be made explicit simply by following the sequence given by the text. Consequently, at the end of the sequence of interpretations, the meaning of the final result is established, thus showing that the result is the one desired. Luckily, we have evidence that, for texts of that kind, some ancient Chinese commentators read proofs of the correctness in this way. However, such texts for algorithms are not specific to China, since texts found in several other scholarly cultures also present the same property. 
Second, the text for an algorithm could prescribe the same operation in different ways: Sometimes, the speech act is carried out directly, designating the operation by a term like "multiplying"; in other cases it is carried out indirectly. I gave two examples of the latter, with the terms "making communicate" or "breaking up." In both cases, the operations were prescribed by terms indicating the intentions motivating their use - in other words, the goal, or the meaning of the result. This constitutes a fundamental similarity in the way in which operations were prescribed indirectly. This feature explains why such texts indicate, in their very formulation, reasons for the correctness of the algorithm described. In fact, there is evidence in our sources supporting this conclusion: reading the ancient commentators on these texts also shows how they develop their proofs of correctness by reading the arguments put forward in this feature of the formulation of the algorithm.

In both types of cases, the commentators handled the texts for the algorithms in specific ways to bring the reasons indicated to light: in the first type, they exploited the structure of the narrative; in the second, they relied on the terms used.

However, despite the fundamental similarity of their indirect prescriptions, the second type of texts analyzed also show key differences. The terms used to indirectly indicate operations in The Nine Chapters captured the meaning of the operation not only at a material but also at a formal level, one at which relationships between various procedures could be established. By contrast, the Book of Mathematical Procedures, apparently composed some two centuries earlier, indirectly prescribed operations by way of their material meaning. If the Book of Mathematical Procedures belonged to the same Chinese written tradition that produced The Nine Chapters and its commentaries, these texts may provide evidence of the emergence of an interest in formal properties in mathematics. I have argued elsewhere that such an interest for formal properties permeated The Nine Chapters and its commentaries. However, it is not perceptible in the Book of Mathematical Procedures.

Despite the differences in how texts for algorithms referred to reasons for correctness, I was led to an unexpected conclusion: Practitioners apparently wanted texts that had this property, to the point that we find distinct types of text realizing it. As to why, I hypothesize that the answer could be found in a result arising from psychological research. Apparently, practitioners using texts of instructions such as algorithms use them all the better when they understand what they are doing. ${ }^{38}$ Hence, to me, the evidence of the texts above shows a constant and stable drive, among practitioners, to shape texts for algorithms that would yield understanding. The two families of text examined above show two main ways in which practitioners achieved this goal. Moreover, the difference between the Book of Mathematical Procedures and The Nine Chapters may even highlight a historical evolution in the ways in which

\footnotetext{
${ }^{38}$ I owe this notion to Jacques Virbel, who took part in research in cognitive psychology on texts of instructions (private communication). Compare also J. Virbel, J.M. Cellier, J.L. Nespoulous (éds.), Cognition, discours procédural, action. Pôle Universitaire Européen de Toulouse \& PRESCOT, Novembre 1997, p. 163; Cognition, discours procédural, action. Volume II. PRESCOT, Mai 1999, p. 308 .
} 
practitioners shaped such texts. In other words, their features simply emphasize that the texts were made and used by human practitioners rather than by machines, as previous historians perhaps surreptitiously assumed.

Cavillargues

Acknowledgments It is my pleasure to express my deepest gratitude to John Holt, who had the difficult task of taming my English, and to Sarah-Jane Patterson who helped me in a crucial way to implement these changes. Without them, the paper would not be as readable as it has become. Nevertheless, I remain responsible for all remaining shortcomings. My most sincere thanks to Gila Hanna and Niels Jahnke, for their support and their patience in all circumstances! 


\section{References}

Chemla, K. (1991). Theoretical aspects of the chinese algorithmic tradition (First to Third Century). Historia Scientiarum, 42, 75-98; Errata in the following issue.

Chemla, K. (1992). Les fractions comme modèle formel en Chine ancienne. In: K. C. Paul Benoit, \& J. Ritter (Eds.) Histoire de fractions fractions d'histoire (pp. 189-207, 405, 410). Basel: Birkhäuser.

Chemla, K. (1996). Relations between procedure and demonstration: Measuring the circle in the "Nine chapters on mathematical procedures" and their commentary by Liu Hiu (3rd century). In: H. N. Jahnke, N. Knoche, \& M. Otte (Eds.) History of Mathematics and Education: Ideas and Experiences (pp. 69-112). Goettingen: Vandenhoeck \& Ruprecht.

Chemla, K. (1997). What is at stake in mathematical proofs from third-century China? Science in Context, 10, 227-251.

Chemla, K. (1997/1998). Fractions and irrationals between algorithm and proof in ancient China. Studies in History of Medicine and Science. New Series, 15, 31-54.

Chemla, K. (2003). Generality above abstraction: the general expressed in terms of the paradigmatic in mathematics in ancient China. Science in context, 16, 413-458.

Chemla, K. (2006). Documenting a process of abstraction in the mathematics of ancient China. In: C. Anderl, \& H. Eifring (Eds.) Studies in Chinese Language and Culture - Festschrift in Honor of Christoph Harbsmeier on the Occasion of his 60th Birthday (pp. 169-194). Oslo: Hermes Academic Publishing and Bookshop A/S.

Chemla, K., \& Shuchun, G. (2004). Les neuf chapitres. Le Classique mathématique de la Chine ancienne et ses commentaires. Paris: Dunod, pp. 27-39.

Cullen, C. (2004). The Suan shu shu 等數書 'Writings on Reckoning': A translation of a Chinese mathematical collection of the second century $B C$, with explanatory commentary. Needham Research Institute Working Papers Vol. 1. Cambridge: Needham Research Institute.

Guo Shuchun郭書春 (1984). 《九章算術》和劉徽注中之率概念及其應用試析 (Analysis of the concept of lï and its uses in The Nine Chapters on Mathematical Procedures and Liu Hui's commentary) (in Chinese). Kejishi Jikan 科技史集刊 (Journal for the History of Science and Technology), 11, 21-36.

Guo Shuchun郭書春 (1992). Gudai shijie shuxue taidou Liu Hui 古代世界數學泰斗劉徽 (Liu Hui, a leading figure of ancient world mathematics), 1st edn. Jinan: Shandong kexue jishu chubanshe.

Høyrup, J. (1990). Algebra and naive geometry: An investigation of some basic aspects of Old Babylonian mathematical thought. Altorientalische Forschungen, 17, 27-69, 262-324.

Li Jimin李繼閔 (1982). Zhongguo gudai de fenshu lilun中國古代的分數理論'. In: W. Wenjun (Ed.) 'Jiuzhang suanshu' yu Liu Hui [The Nine Chapters on Mathematical Procedures and Liu Hui] (pp. 190-209). Beijing: Beijing Shifan Daxue Chubanshe.

Li Jimin李繼閔 (1990). Dongfang shuxue dianji Jiuzhang suanshu ji qi Liu Hui zhu yanjiu 東方數學典籍——《章算術》及其劉徽注研究 (Research on the Oriental mathematical Classic The Nine Chapters on Mathematical Procedures and on its Commentary by Liu Hui), 1 Vol. Xi'an: Shaanxi renmin jiaoyu chubanshe.

Li Yan 李㒤 (1958). Zhongguo shuxue dagang. Xiuding ben 中國數學大綱 (Outline of the history of mathematics in China. Revised edition), 2 Vols. Beijing: Kexue chubanshe.

Peng Hao 彭浩 (2001). Zhangjiashan hanjian «Suanshushu»zhushi

張家山漢簡《算術書》注釋(Commentary on the Book of Mathematical Procedures, a writing on bamboo slips dating from the Han and discovered at Zhangjiashan). Beijing: Science Press (Kexue chubanshe).

Qian Baocong 錢寶琮 (1964). Zhongguo shuxue shi 中國數學史 (History of mathematics in China). Beijing: Kexue chubanshe.

Rashed, R. (2007). Al-Khwarizmi. Le commencement de l'algèbre. Texte établi, traduit et commenté par R. Rashed. Sciences dans l'histoire. Paris: Librairie scientifique et technique Albert Blanchard. 
Wu Wenjun 吳文俊 (Ed.) (1982). 'Jiuzhang suanshu' yu Liu Hui 九章算術與劉徽 [The Nine Chapters on Mathematical Procedures and Liu Hui]. Beijing: Beijing Shifan Daxue Chubanshe.

Wu Wenjun 吳文俊, Bai Shangshu 白尚恕, Shen Kangshen 沈康身, LI Di 李迪 (Eds.) (1993). Liu Hui yanjiu 劉徽研究 (Research on Liu Hui). Xi'an: Shaanxi renmin jiaoyu chubanshe, Jiuzhang chubanshe. 